\title{
Leveraging Area Bounds Information for Autonomous Multi-Robot Exploration
}

Tsung-Ming Liu

Fordham University

Damian M. Lyons

Fordham University

Follow this and additional works at: https://fordham.bepress.com/frcv_facultypubs

Part of the Robotics Commons

\section{Recommended Citation}

Liu, Tsung-Ming and Lyons, Damian M., "Leveraging Area Bounds Information for Autonomous Multi-Robot Exploration" (2014). Faculty Publications. 38.

https://fordham.bepress.com/frcv_facultypubs/38 accepted for inclusion in Faculty Publications by an authorized administrator of DigitalResearch@Fordham. For more information, please contact considine@fordham.edu. 


\title{
Leveraging Area Bounds Information for Autonomous Multi-Robot Exploration
}

\author{
Tsung-Ming Liu and Damian M. Lyons \\ Fordham University, New York NY 10105, USA \\ \{tliul7, dlyons\} @ fordham.edu
}

\begin{abstract}
In this paper we propose an approach, the Space-Based Potential Field (SBPF) approach, to controlling multiple robots for area exploration missions that focus on robot dispersion. The SBPF method is based on a potential field approach that leverages knowledge of the overall bounds of the area to be explored. This additional information allows a simpler potential field control strategy for all robots but which nonetheless has good dispersion and overlap performance in all the multi-robot scenarios while avoiding potential minima. Both simulation and robot experimental results are presented as evidence.
\end{abstract}

Keywords: Multi-robot exploration, potential field path-planning, autonomous systems.

\section{Introduction}

A common autonomous robot application is to send out multiple robots to an unknown environment to explore and collect information about the environment. For example, in a C-WMD (counter weapon of mass destruction) mission the robot mission designer may know there is a bomb in the building and we want to send out robots to search for the bomb. In this case, the critical point of the mission is to cover as much of the area within the building as possible in the shortest time. Or, as Baxter [1] suggested, in some scenarios where the environment is hazardous and a search and rescue mission needs to be carried out, we can send a team of robots to search for survivors; such missions are time-critical and detailed environment information is not needed, rather a fast, effective scan with the on-board sensors is much more crucial for the mission. In addition, if multiple robots are being used to increase exploration efficiency, then maximizing dispersion and minimizing overlap in scanning are particularly important. For simplicity and efficiency, it is preferred to have the robots depend and communicate with each other as little as possible, and for the control strategy of the robots to be as uniform as possible.

We propose the following as reasonable and useful assumptions to make about the mission: (1) the robot mission designer knows something about the environment, but not everything. For instance, the rough dimension of the building to be searched is known but not the indoor floor map details. And (2) some inter-robot communication is possible, but only a minimum amount of data transfer is preferred.

In this scenario we would like to have a simple and uniform approach to the control of robot team members: easy to synchronize and communicate between robots, and coordinate exploration strategy, without massive transfer of data or environment information collected by the robots. 
In [2], Ozisik et al. described an Occupancy-Grid based SLAM method, however it was not optimized for multiple robot efficiency. In [3] Birk shows how to merge occupancy grid maps from multiple robots, and as does Herath [4]. However they focus on feature-recognition and merging data from multiple robots. Lyons et al [5] discuss gridbased methods to combine maps so as to avoid transitory readings from other robots.

Arkin [6] presents a potential field approach to goal-directed robot motion, using noise and spin fields to address minima issues. Baxter [1] also presents a potential field approach in which the field is shared among robots. Cepeda [7] proposed a behavior based approach for multi-robot exploration, but such an approach has difficulty in robot exploration status synchronization. Popa [8] uses potential fields for dispersion of sensor networks, but doesn't discuss exploration or path-planning. Batalin [9] presents an approach with good dispersion results but is not as strong on path-planning and searching. Min [10], Mi [11] and Schwager et al. [12] all provided novel topological approaches for dispersion and coverage of multi-robot exploration, and Jenson [13] proposed using wireless signals for the same purpose; however the detailed path-finding strategy is not part of the solutions.

Renzaglia [14] and Julia et al. [15] use the potential field method for decentralized robot control, and a frontier-based leader-follower approach for breaking out of potential field minima. In this approach, follower robots evaluates explored area, and the position of the other robots and try to move to the frontier explored region, and the leader robots are unbounded by the other robots, solely focusing on exploration. As a result, communication and calculation of information shared between the robots, as well as transition between roles of leader and follower become crucial and risk failure if any link in this cooperation failed.

In this paper we also propose an approach using potential fields to drive the robots to unexplored areas, and so [14] is a natural point of comparison. However, we don't require an additional, leader-follower class of robot to handle the impact of potential minima. Furthermore, we integrate sensor direction and range into the model, and introduce coverage into the potential field calculations, resulting in a more realistic model with a relatively simple control strategy.

In Section 2 we will discuss the problems outlined above and propose our improved approach, the Space-Based Potential Field (SBPF) method, to solve the issues. In Section 3 and 4 we will use the proposed approach in simulation and real-world robot experiments, examine and verify its efficiency in comparison with the leader-follower approach. Lastly in Section 5 we discuss the result and future of the strategy.

\section{Problem Formulation}

In this section we present our approach to the multi-robot dispersion and exploration problem by leveraging knowledge of the overall area bounds. The algorithm must be able to disperse the robots for the exploration mission and also drive the robots to unexplored areas. Keeping robots dispersed will ensure that they exhibit better coverage by decreasing overlap in scanning while the whole map is explored.

\subsection{Potential Field Approach and Local Minimum Issue}

The potential field approach [6] has been used for directing robot motion towards a goal while avoiding obstacles. For our purposes, the unexplored area needs to act as an attractive goal for robots. We will not model the unexplored area as a frontier $[14,15]$ 
but will rather have the unexplored area itself exert an attractive force. Because we assume we know the overall bounds of the area, we know the maximum area to be explored. This method supports robot dispersion, since each robot sees the other robots as moving obstacles and tries to steer clear of them. We can now just use random noise to avoid local minimum issues.

\subsection{Potential Level}

Slightly different from previous versions of the potential field methods, our approach divides the map into multiple grids/cells where each cell has a potential level, representing the level of exploration or scanning by sensors. As the robot sensor covers the area once or more, the potential level will increase.

The potential level is used for avoiding obstacles; if the sensor detects an obstacle, it will increase the potential level of the associated cell. In addition, the potential level increases when the region has been visited by the robots, or covered by any robot sensors.

Lastly the potential level will guide the robots to unexplored areas. As time increases, cells with low or zero potential level will generate more attractive potentials, and will pull the robots toward them for sensor readings.

\subsection{Avoiding Expl ored Areas Using Repul sive Potential}

Repulsive potential is generated by the obstacles, or grid cells with obstacle potential level, and all such cells have the same magnitude of repulsion. However, the direction of the repulsive vector is perpendicular to the direction from the obstacle to the robot:

- Repulsive potential given by previously visited or scanned areas

- Repulsive potential given by the other robot(s)

- Repulsive potential given by the obstacles, or cells marked as obstacles. The magnitude is the same, but the direction is rotated by 90 degrees.

Note that the difference in direction for obstacle repulsion: the result here is that instead of direct repulsion from the obstacle, it will form a spin field around the obstacle [6], creating a smoother route away from the obstacle. Furthermore when the robot is near a symmetric shaped obstacle that generates equally repulsion potential on both sides, the robot will go around the obstacle instead of trapped in an equilibrium state.

Modifying [14], we define the repulsive potential field to be given by

$$
U_{\text {rep }}\left(q, q_{i}\right)= \begin{cases}\frac{1}{2 h_{\text {rep }}(c)}\left(\frac{1}{\rho(q)}-\frac{1}{\rho_{0}}\right)^{2}, & \rho(q) \leq \rho_{0} \\ 0 & , \rho(q)>\rho_{0}\end{cases}
$$

where $q$ is the robot location, $q_{i}$ is the potential level of each other cell in the map grid, $\rho_{0}$ is the maximum sensing distance of the robot, and $\rho(q)=\left\|q-q_{i}\right\|$.

The function, $h_{r e p}(c)$ gives us a repulsive variable factor for this specific time of coverage, $c$, as follows

$$
h_{\text {rep }}(c)=c k_{\text {rep }}
$$


The use of the coverage factor in the equation makes the repulsive force get larger with smaller coverage and smaller as coverage grows. At the beginning of the exploration, the robot will be more likely to be driven away from previously explored areas as well as the other robots.

The gives us the repulsive force, for each cell, as

$$
F_{r e p}\left(q, q_{i}\right)=\left\{\begin{array}{l}
\frac{1}{2 h_{r e p}(c)}\left(\frac{1}{\rho(q)}-\frac{1}{\rho_{0}}\right) \frac{q-q_{i}}{\rho^{3}(q)}, \rho(q) \leq \rho_{0} \\
0 \quad, \rho(q)>\rho_{0}
\end{array}\right.
$$

Lastly, the summation of the repulsive force from each other cell with a potential level gives the robot at this particular location a repulsive force that drives it away from obstacles and previous visited areas.

$$
F_{r e p}(q)=\sum_{i=1}^{n} F_{r e p}\left(q, q_{i}\right)
$$

\subsection{Attraction to Unexpl ored Areas}

When a cell in the occupancy map has zero potential level, it is then determined than this area has not been scanned nor visited by any robots, and contributes an attractive potential to the potential field. It essentially means that this block of map has not been visited nor sensor scanned, and for this reason it should be more attractive to robots.

This approach differs from an approach that models an unexplored frontier, since it treats every unexplored cell as an attractive potential goal. Because in this method an unexplored frontier perimeter does not need to be maintained or calculated, this approach is less demanding computationally. Of course, being are a based, it can have larger memory requirement from the beginning since the entire map, though empty, is present in memory from the start for calculation. And as we will see presently, we do need to store some perimeter information.

The attractive potential field, again after [14], is

$$
U_{a t t}\left(q, q_{i}\right)=\frac{1}{4} h_{a t t}(c) \rho_{g o a l}^{4}\left(q_{i}\right)
$$

The function, $h_{\text {att }}(c)$ gives us an attractive variable factor for this specific time of coverage, $c$. And once again we define

$$
h_{a t t}(c)=k_{a t t} /_{c}
$$

Where the use of the coverage factor in the equation make the attractive force get larger with smaller coverage and smaller as coverage grows.

The attractive force is given by 


$$
F_{a t t}\left(q, q_{i}\right)=h_{a t t}(c)\left(q_{g o a l}-q\right) \rho_{g o a l}^{2}
$$

And summing up all the attractive cells we can get the sum attractive force applied to the specific robot,

$$
F_{a t t}(q)=\sum_{i=1}^{n} F_{a t t}\left(q, q_{i}\right)
$$

\subsection{Sum of forces}

This summation of force will attempt to drive the robot away from the other robots, obstacles and previously visited areas, toward unexplored areas.

$$
\begin{aligned}
F_{\text {total }}= & F_{\text {rep,explored area }}+F_{\text {rep,other robots }}+ \\
& F_{\text {rep,obstacles }}+F_{\text {att,unexplored area }} \\
& =m \ddot{q}-v \dot{q}
\end{aligned}
$$

where $m$ is the mass of the simulated robot and $v$ a viscous damping factor as in [14].

Due to the coverage factor in the repulsion and attraction force, the net result is that in the beginning of an exploration mission where the map coverage is small, the robot will tend to focus on spreading out or moving away from the explored area (if applicable, say the map is already $20 \%$ explored by the previous robot and this one just joined the mission), while at a later stage of the mission, the coverage control factor will focus on drawing the robots to un-explored areas, and fill up the empty sections on the map.

\section{Simulation Results}

In this section we present simulation results for the SBPF method using building maps similar to those of Renzaglia [14] so that side-by-side performance comparisons can be made. However, unlike [14], we will assume that the robots are equipped with laser sensors that have cone-shaped field of view constraint of angle $\alpha \leq \pi$.

We measure the coverage over time as a metric of performance. If $c(t)=\alpha$ is the coverage at time $t$, then we define the performance to reach that coverage as $P_{\alpha}=1 / t$.

In the case where more than one robot is being used, then we each define the speedup for $c(t)=\alpha$, by analogy with parallel computing, as the performance of the $n$ robot case divided by that of the one robot case: $S_{n, \alpha}=P_{n, \alpha} / P_{1, \alpha}$. Finally, we can define efficiency as $E_{\alpha}=P_{n, \alpha} /\left(n P_{1, \alpha}\right)=S_{\alpha} / n$. We will adopt a coverage of $\alpha=95 \%$ as our test coverage.

We will look at performance on three examples. In the first case, an empty room, we focus on showing the SBPF performance for 1 to 3 robots. In the second two cases, more complicated maps, we focus on the comparison of the SBPF multirobot $(n=3)$ performance with the leader-follower method [14].

\subsection{Open space example}

The first example uses a map of an empty building. This example shows how the algorithm directs the robot team to spread out and head toward unexplored areas. 
Figure 1 shows three snapshots from the execution of the SBPF exploration of the map with three robots. In Figure 1 (left) the arrows indicate the direction of potential field at each point in space (i.e., at that cell in the occupancy grid), the thick black lines show the regions that robot sees as obstacles or walls. Each robot is indicated by grey dot, the shaded cone-shapes indicate the sensor-sensing region for each robot, and the darkness of the shade of each cells represents the value of the potential level for that cell, where a darker cell means higher potential level values. If the map is mostly explored then most cells will have been heavily shaded. All of the robots start at lower left corner in this example.
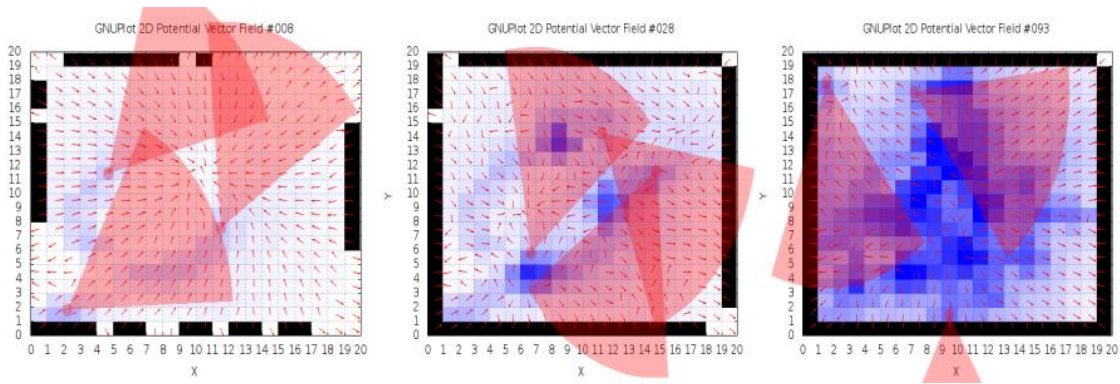

Three snapshots of the occupancy grid information for three successive time steps $(\mathrm{t}=8,28$, 93) during the exploration of the empty room map with three robots using the SBPF method.

Figure 1. Exploration of the empty room map. (100 steps)

In Figure 1 (bottom left) we see the robots successfully spread out at the final stage of exploration. In addition to the robots dispersion in location, the robots also have different headings and have non-overlapping sensing area, which will increase the explore efficiency compare to sensing region overlapping.

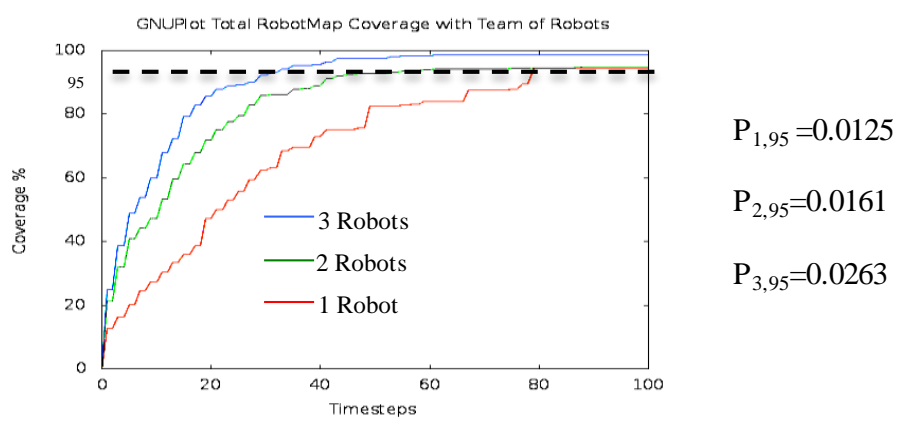

Figure 2. Graph of percentage explored area versus time-steps with one robot (bottom), two robots (middle) and three robots (top) for the empty map using the SBPF method.

From the graph of percentage of explored area versus time (Figure 2), we can see that the percentage reaches $100 \%$ explored without deadlock or encountering potential equilibria. The speedup for the two robot case is $S_{2,95}=1.29$ and for three robots is $S_{3,95}$ $=2.1$. The efficiency is relatively constant for both: $E_{2,95}=0.65$ and $E_{3,95}=0.7$.

This shows that the exploration efficiency increased with number of robots, and this cannot be achieved without robot team dispersion and avoidance of sensory overlapping. 
Otherwise, multi-robot exploration will not achieve better efficiency than single robot exploration.

\subsection{Two Room Example}

In this next example (Figure 3), the robots need to go in and out of two rooms in order to explore the building fully. This example is one that can give rise to potential field minima between the two rooms. Also, because each room is surrounded by the walls, the net repulsion from the walls outside of the room will form a force preventing a robot from entering the room. In addition, once the robot is inside the room, the net repulsion from the surrounding easily trap a robot inside.
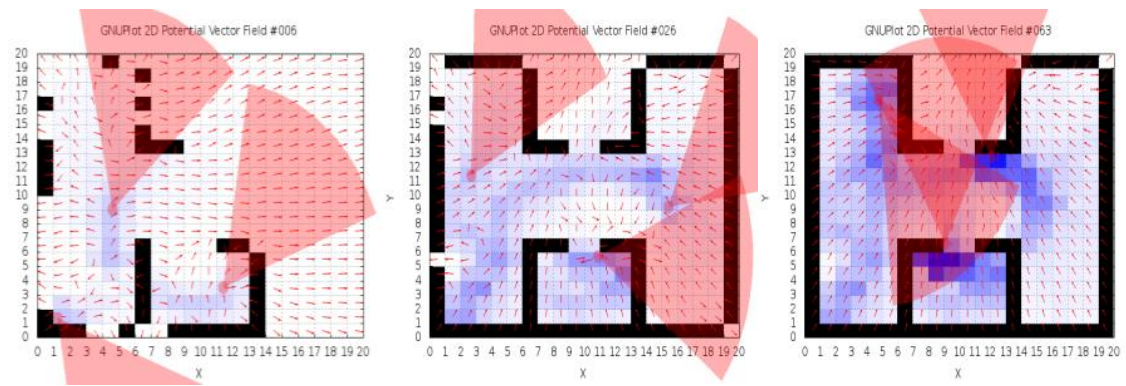

Three snapshots of the occupancy grid information for three successive time steps $(t=6,26,63)$ during the exploration of the two room map with three robots using the SBPF method.

Figure 3. Exploration of the two room map.

In this example (Figure 3, top right and bottom left) we can see the robots first reach the end of the hallway, then come back and go into the room (surrounded by walls) where initially they were not able to go. The results indicate that using our algorithm the robots successfully averted any equilibrium issues. The robots successfully enter and exit the room, without becoming stuck in a potential field minimum between the walls, and eventually achieve $100 \%$ map coverage (Figure 4).

Figure 4 compares the multirobot ( $n=3$ ) performance of the SBPF approach (top left) with that of the leader-follow approach [14] (top right) and calculates the performance metrics for each (bottom). We can see the difference between the two methods regarding both how the robots escape potential minima and how unexplored areas attract the robots. In the leader-follower approach, Figure 4 (top right), we first see a slow growth in coverage, where the robots are attracted by the closest frontier region, the boundary between explored areas and unexplored areas. Therefore, when the robots travel by a doorway in the map, the unexplored area in the room beyond will draw them before reaching the end of the doorway. When one of the (follower) robots entered the doorway, it was caught in a potential minima, slowing coverage, marked as (1) of Figure 4 (top right), but shortly was pulled out by the leader robot.

With the SBPF approach, the robots are more attracted by the larger unexplored areas than the smaller ones. Therefore the robots tend to explore the other side of the room as shown in Figure 4 (topleft) which results in the faster growth in coverage percentage marked (1) on that graph. For smaller coverage (60\% and $80 \%)$, the SBPF method shows a speedup of 1.9 and 1.45 over the leader follower approach, principally because of this. 

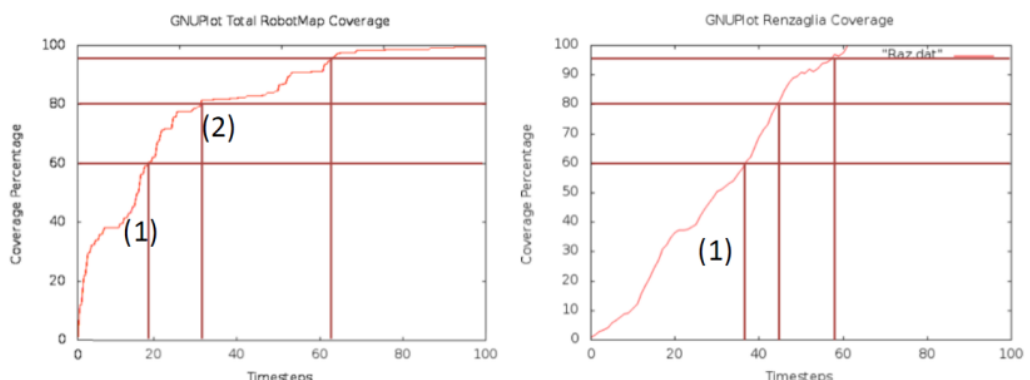

\begin{tabular}{llc} 
Coverage \% & SBPF & Leader-Follower \\
\hline $95 \%$ & $\mathrm{p}_{3,95}=0.016$ & $\mathrm{p}_{3,95}=0.017$ \\
$\mathbf{8 0 \%}$ & $\mathrm{p}_{3,80}=0.032$ & $\mathrm{p}_{3,80}=0.022$ \\
$\mathbf{6 0 \%}$ & $\mathrm{p}_{3,60}=0.052$ & $\mathrm{p}_{3,60}=0.027$ \\
\hline
\end{tabular}

Figure 4. Performance for the two-room map. (100 steps)

After that, the robots return and finish the smaller unexplored areas, which is the two small rooms. They have to travel through previously explored areas, therefore the map coverage percentage does not increase in the time between, marked (2) in Figure 4 (top left). Both approaches perform similarly towards the end of coverage $\left(P_{95}\right)$, despite the fact that the proposed approach has in fact a more realistic (and hence constrained) sensor model.

\subsection{Obstacle example}

In the example in Figure 5 the robots will attempt to reach the other side of abuilding that has several internal obstacles and walls including a permanently enclosed area.
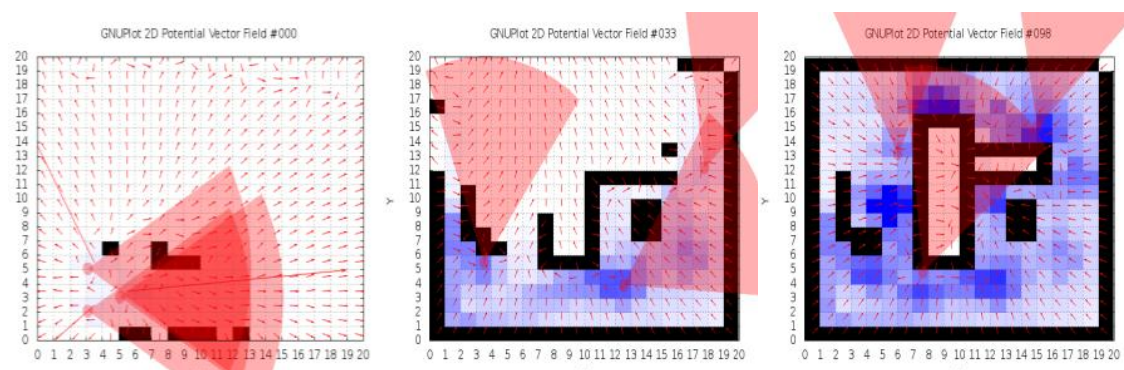

Three snapshots of the occupancy grid information for three successive time steps $(t=0,33$, 98) during the exploration of the room with obstacles map with three robots using the SBPF method.

Figure 5. Exploration of athe room with obstacles map.

In our simulation, the line of sight of the robot sensors will be blocked by obstacles, and cannot therefore cover the region beyond them. As a result, regions completely occluded by obstacles or walls will always stay unexplored. As the algorithm stands, these regions become an attractive potential source as time goes by: impossible to explore, but 
distracting the robots from exploring other areas. Unless this is addressed, the performance of the SBPF method would be much worse for these scenarios that the leaderfollower approach!

This issue is addressed by 'zeroing-out' any regions that are found to be enclosed. If walls or obstacles completely surround a region (as determined by examining the state of the occupancy map), its interior cells are then treated as obstacles and are no longer part of attractive potential field.

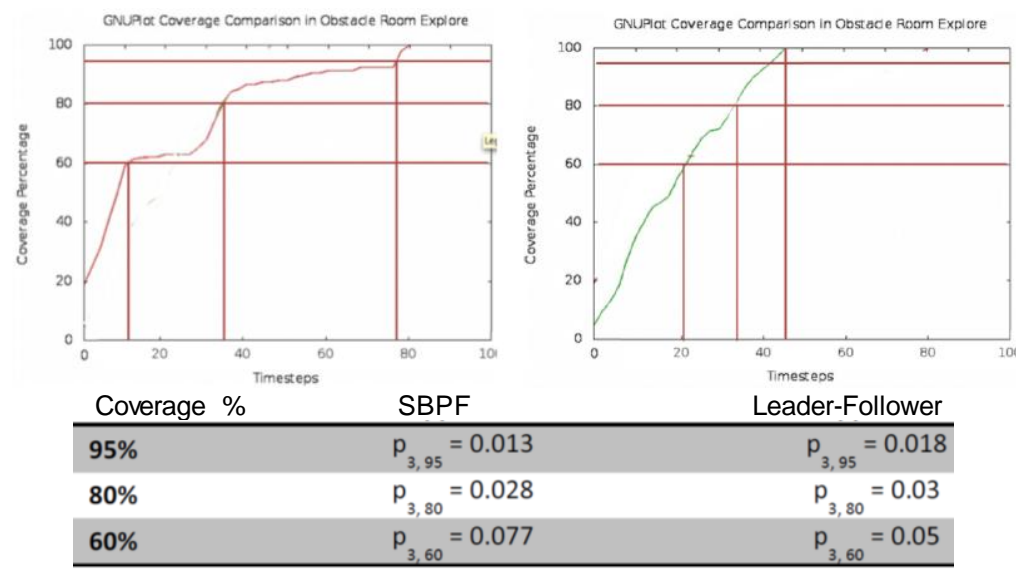

Figure 6. Performance for the map with obstacles. (100 steps)

Figure 6 shows the comparison of the SBPF method with this extension and the leader-follower approach for a room with enclosed obstacles. Overall, coverage goes up in a linear rate at the early stage of the exploration mission, as the robots aim for the larger blank areas. The rate then decreases as obstacles are encountered and occlude sensing (more so for the SBPF method with its more realistic constrained field of view assumption), and recovers as the robots clear the occlusion.

The effect of the extension is visible in the coverage graph of Figure 6 (top left) as a series of steps. We can see the robots complete coverage (the enclosed area cannot be explored) without getting stuck between the obstacles or distracted by the enclosed area. Furthermore the overall performance is now similar to that of the leader-follower approach as shown in Figure 6 (bottom). Though even in this case, we see that the SBPF covers more quickly early in coverage (the $P_{3,60}$ results show a speedup of 1.54 ).

\section{Robot Experiments}

In this section we show that our method transfers well from simulation to real implementation. We describe results from carrying out this algorithm with a team of two Pioneer 3-AT robots.

\subsection{Environment Setup}

The room to be explored is 5 meters by 5 meters, arranged as shown in Figure 7 . The partition is placed to block the robot's line of sight; complete coverage can not be attained unless the robots move into the enclosed area. 


\subsection{Robot Setup}

We use two Pioneer AT-3 robots each with pre-installed ARIA library control and SICK LMS-200 laser sensor with 180 degrees sensing field of view and $10 \mathrm{~m}$ sensing range.
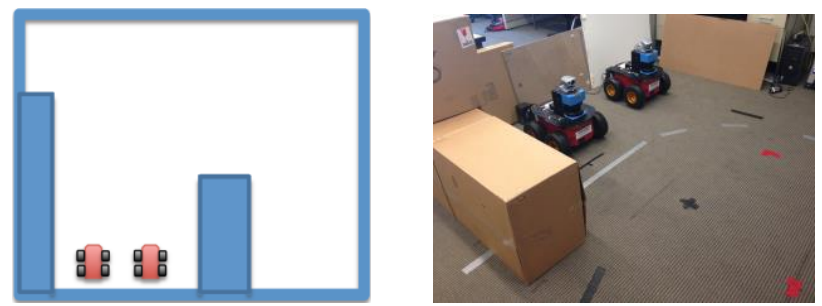

Figure 7. Experimental map and robot setup

The Pioneer 3-AT implementation differs from the simulation in one important aspect: The robot takes its motion direction from the potential field, and the magnitude depends on both the potential force magnitude and direction. However, when the direction points directly behind the robot, then the robot will reverse and back off in addition to turning away.

The robots are allowed to move at $1 \mathrm{~meter} / \mathrm{second}$ maximum velocity, and will slow down or stop if another object is in the path of movement. The program terminates normally if the map is $90 \%$ explored, and terminates in failure if any robot stays in the same position for more than 10 seconds due to collision with robots or obstacles.

\subsection{Experiment Result and Analysis}

An illustrative example experimental result is shown in Figure 8 below. The team of two robots reaches $90 \%$ coverage of the 4 by 4 meter room with obstacles within 30 seconds in 5 out of 10 times (Figure 9). In 2 of 10 times, this took 50 seconds, and the remaining 3 ended in failure due to robot collision.

The motion of robots entering and exiting of the obstacle-surrounded area (see Figure 8 , lower left region) results in frequent collisions. This is likely to be caused by the latency and frequency of the robot information packet transmission: the algorithm determines that a region has not yet been explored, forms a source of attractive potential and sends robots toward that direction, but at the same time the potential field cannot predict that both robots will move toward the same region. The robots will collide if they do not update their location and sensor readings frequently. This indicates an important future direction of research for this work. We will return to this topic when we discuss the next steps for this work in Section 5.

Robot interference with each other's sensor readings is another cause of error. No filtering was added to eliminate these mutually interfering sensor readings (e.g., as in [5]) and to record a robot as a permanent obstacle with appropriately high repulsive effect. 

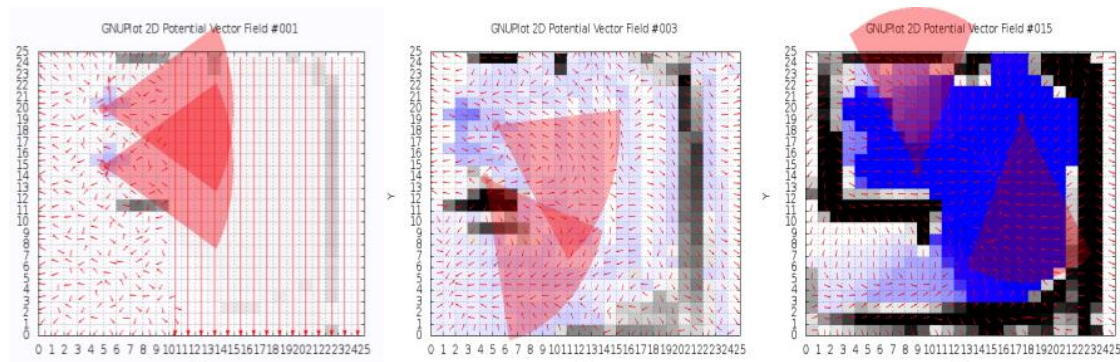

Three snapshots of the occupancy grid information for three successive time steps $(t=1,3,15)$ during the exploration of the lab map using two Pioneer 3-AT robots.

Figure 8. Exploration using two Pioneer 3AT robots.

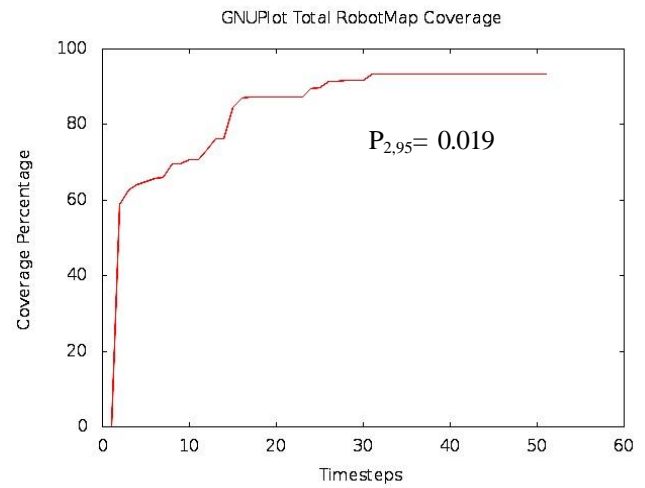

Figure 9. Percentage covered over time chart. (40 Seconds)

\section{$5 \quad$ Conclusion and Future Work}

In this paper we have presented a novel approach to motion planning for multi-robot search problems, the Space-Based Potential Field (SBPF) method. We make the assumption that the overall size of the area to be searched is known and we use this information to develop a simple, uniform potential field strategy that has good dispersion/overlap characteristics and avoids local minima.

We present several simulation results that show the performance of the method. We compare the performance on several maps with that of Renzaglia's [8] leader-follower approach. We use metrics of performance, speedup and efficiency based on similar parallel computation metrics. The proposed approach has a simpler, uniform control strategy and incorporates more realistic sensing assumptions. We show that nonetheless it performs no worse on these examples that the more complicated leader-follower approach, and in some cases performs much better. In particular the approach has strong early coverage performance compared to the leader-follow approach on these maps.

We also show results from an implementation of the SBPF method on a team of two Pioneer 3-AT robots exploring a small area. This demonstrates that the simulation results will transfer to practice and also points to future work to improve the method. The 
performance of the robot team was less robust than the simulation, working in 7 out of 10 trials. The current method does not address the issue of the decentralized control architecture, and this was the principal cause of error when we reduced our simulation to practice.

Our work in progress now is studies the robot communication aspect of this problem in more detail. The objective is to develop efficient approaches to transmitting and sharing the map information, including filtering out the mutual interference effects of many robots operating in close proximity, as discussed in [5].

\section{Acknowledgement}

This research was supported by the Defense Threat Reduction Agency, Basic Research Award \#HDTRA1-11-1-0038

\section{References}

[1] J. L. Baxter, E. K. Burke, J. M. Garibaldi, and M. Norman, "Multi-Robot Search and Rescue: A Potential Field Based Approach" in Autonomous Robot and Agents, vol. 76, Springer Berlin Heidelberg, 2007, pp. 9-16.

[2] O. Ozisik and S. Yavuz, "An Occupancy Grid Based SLAM Method" in 2008 Intl. Conf. CIMESA, pp. 117-119.

[3] A. Birk and S. Carpin, "Merging Occupancy Grid Maps from Multiple Robots" in Proc. IEEE, vol. 94, pp. 1384-1397

[4] D. C. Herath, S. Kodagoda, and G. Dissanayake, "New Framework for Simultaneous Localization and Mapping: Multi map SLAM" in Proc. 2008 IEEE Intl. Conf. Robotics Automation, pp.1892-1897

[5] D. M. Lyons, K. Shrestha, and T. Liu, "Fusion of Ranging Data from Robot Teams Operating in Confining Areas" in Proc. SPIE Defense, Security Sensing; Multisource, Multisensor Information Fusion, Baltimore MD April 2013

[6] R. C. Arkin, "Behavior-Based Robotics", Denver CO: Bradford Publishing, 1998, pp. 100-110

[7] J. S. Cepeda, L. Chaimowicz, R. Soto, J. L. Gordillo, E. A. Alanise-Reyes, and L. C. Carrillo-Arce, "A Behavior-Based Strategy for Single and Multi-robot Autonomous Exploration" in 2012 IEEE Sensor J., vol. 12, pp. 12773-12797

[8] D. O. Popa, C. Helm, H. E. Stephanou, and A. C. Sanderson, "Robotic Deployment of Sensor Network Using Potential Fields", in Proc. 2004 IEEE Intl. Conf. Robotics Automation, pp. 642-647

[9] M. A. Batalin and G. S. Sukhatme, "Coverage, Exploration and Deployment by a Mobile Robot and Communication Network”, in Proc. 2003 Intl. Workshop on Info. Processing Sensor Networks, pp. 2003

[10] H. J. Min and N. Papanikolopoulos, "The Multi-robot Coverage Problem for Optimal Coordinated Search with an Unknown Number of Robots", in Proc. 2011 IEEE Intl. Conf. Robotics Automation, pp. 2866-2871

[11] Z. Mi, Y. Yang, and G. Liu, "Coverage Enhancement of Mobile Multi-agent Networks while Preserving Global Connectivity" in Proc. 2011 IEEE Intl. Conf. Robotics Automation, pp. 5381-5386

[12] M. Schwager, D. Rus, and J. Slotine, "Unifying Geometric, Probabilistic, and Potential Field Approaches to Multi-robot Coverage Control", in 2011 Intl. Symposium ISRR, pp. 21-38

[13] E. Jensen and M. Gini, "Rolling Dispersion for Robot Teams", in Proc. 2013 Intl. Joint Conf. Artificial Intelligence, pp. 2473-2479

[14] A. Renzaglia and A. Martinelli, "Potential Field Based Approach for Coordinate Exploration with a Multi-robot Team", in 2010 IEEE Intl. Workshop on Safety Security Rescue Robotics, pp. 1-6

[15] M. Julia, A. Gil, L. Paya and O. Reinoso, "Potential Field Based Integrated Exploration for Multi-robot Teams", in Proc. 2008 Intl. Conf. Informatics Control, Robotics Automation, pp. 308-314 